\title{
Monitoring of lonosphere and Magnetosphere Plasma and High Energy Charge Particle Fluxes in Multi-satellite Measurements in Wide Range of Altitudes
}

\author{
Vladislav Osedlo ${ }^{1}$, Oleg Abanin ${ }^{1}$, Natalia Vlasova $^{1}$, Vladimir Kalegaev ${ }^{1,2}$, Nikolay Pavlov ${ }^{1}$, \\ Vasilii Petrov ${ }^{1}$, Mikhail Podzolko ${ }^{1}$, Ilia Rubinstein ${ }^{1}$, Sergey Svertilov ${ }^{1,2^{*}}$, Vladimir Tulupov ${ }^{1}$ \\ and Andrey Shemukhin ${ }^{1}$ \\ ${ }^{1}$ M.V. Lomonosov Moscow State University, D.V. Skobeltsyn Institute of Nuclear \\ Physics, 119991, Leninskie Gory 1(2), GSP-1, Moscow, Russia \\ 2 M.V. Lomonosov Moscow State University, Physics Department, 119991, Leninskie Gory, \\ Moscow, Russia
}

\begin{abstract}
A system for monitoring the radiation parameters of near-Earth space is described. This system is based on the multi-satellite measurements made on spacecraft Meteor, Electro, Arktika launched into orbits with a wide range of altitudes. The main instrument for space radiation monitoring is spectrometer of electrons and protons SKIF. Such instruments operate in all spacecraft of mentioned above series. The results of observations of different events connected with solar and geomagnetic activity in 2017 and 2021 years are presented and discussed.
\end{abstract}

\section{Introduction}

Near-Earth space (NES) contains charged particles of ionospheric and magnetospheric plasma, energetic particles of the Earth's radiation belts (ERB), solar and galactic cosmic rays (SCR and GCR) in a wide range of energies [1]. They can lead to damage and failure of electronic equipment installed on spacecraft (SC), can cause disruption of short-wave communications in high-latitude regions, lead to failures in navigation systems and reduce the accuracy of global navigation and positioning systems [2].

Within the framework of multi-satellite measurements on spacecraft launched into orbits with a wide range of altitudes (Meteor - $830 \mathrm{~km}$, Arktika - an elliptical orbit with apogee and perigee heights of $\sim 39$ thousands $\mathrm{km}$ and $\sim 600 \mathrm{~km}$, Electro - geostationary in the plane equator - 36 thousands $\mathrm{km}$ ), for several years at the Skobel'tsyn Institute of Nuclear Physics of Moscow State University (SINP MSU), monitor observations of ionospheric and magnetospheric plasma and energetic electron and proton fluxes have been carried out and continue (spacecraft Arktika-M1). The picture becomes more complete with the involvement of information from identical measuring instruments. Such an instrument, developed and manufactured at the SINP MSU, is a space radiation spectrometer SKIF for

* Corresponding author: sis@,coronas.ru 
measuring fluxes, energy spectra, temporal variations and angular distribution of ERB protons and electrons, SCR particles from solar flares under their combined effect.

Obtaining a large volume of regular monitoring data on the radiation situation in the NES on the basis of multi-tiered measurements allows studying its short- and medium-term dynamics, its relationship with solar activity, is a necessary condition for refining empirical and for creating prognostic dynamic models of space weather, for formation a threedimensional real time dynamic picture of radiation distribution in the ionosphere and the inner magnetosphere.

In this article, a description of the SKIF equipment will be given and examples of the use of complex multi-satellite measurements for the analysis and diagnostics of the radiation state of the NCP will be given.

\section{Observations}

\subsection{SKIF instrument}

The SKIF instrument, developed and created at SINP MSU is intended for monitoring the radiation environment in NES and provides detection of charged particle fluxes (electrons and protons) in a wide energy range from $0.05 \mathrm{keV}$ to more than $160 \mathrm{MeV}$. The SKIF instrument consists of telescopes based on several semiconductor detectors and one scintillation, gas-discharge counters and spectrometers of low-energy electrons and protons based on electrostatic analyzers. The spectrometer of energetic particle, so-called SEP unit of the SKIF instrument provides measurements of the spectra of protons and electrons in the energy ranges of $2-160 \mathrm{MeV}, 0.15-10 \mathrm{MeV}$, respectively. The SEP unit logic forms several energy intervals for particles of each type, i.e. 6 intervals for electrons and 12 for protons. Measurements of the spectra of low-energy electrons and protons in the energy range $0.05-20 \mathrm{keV}$ are performed by electrostatic analyzers (ESA) of electrons and protons (units SGMTD-E and SGMTD-P). The entire recorded energy range $0.05-20 \mathrm{keV}$ ESA of both electrons and protons is divided into 12 intervals. The SKIF instrument includes two identical units duplicating each other's work, but installed in mutually normal directions to study the pitch-angle distribution of particles. The SKIF instrument also includes two identical gas-discharge detector units, in which the SBM20-1 detectors are used. Its readings are used as reference values of fluxes in the upper energy ranges. The detectors are located behind the 2-mm protection of the instrument front wall, normally to the axes of view of the remaining detecting units.

At present, the SKIF instrument operates on three types of Russian SC, i.e. Meteor, Arktika and Electro series. Meteor SC is a polar sun-synchronous satellite with a circular orbit at an altitude of $\sim 830 \mathrm{~km}$ with an orbital period of $\sim 110 \mathrm{~min}$. SC Arktika has an elliptical orbit with apogee and perigee heights of $\sim 39$ thousand $\mathrm{km}$ and $\sim 600 \mathrm{~km}$, respectively, and an orbital period of $\sim 8$ hours. SC Electro is a geostationary satellite located in the plane of the geographic equator at an altitude of $\sim 36000 \mathrm{~km}$. This constellation of satellites makes it possible to effectively monitor radiation conditions in near-Earth space.

\subsection{Experimental data}

The Center for Operational Space Monitoring Data (COSMD) [http://smdc.sinp.msu.ru] has been established at the SINP MSU. The COSMD computer complex carries out storage and processing of experimental data, monitoring and forecasting of space weather parameters 
[4]. The figures below were constructed and can be reproduced on the pages of the COSMD website [http://swx.sinp.msu.ru/tools/ida.php?gcm=1].

For a comparative analysis of the proton and electron flux dynamics according to the data from the SKIF instrument, we also used the experimental data on the electron fluxes from the GOES satellite [http://goes.gsfc.nasa.gov/], on the solar wind (SW) velocity and the interplanetary magnetic field (IMF) with the ACE spacecraft [http://www.srl.caltech.edu/ACE/]. The GOES satellite is a geostationary satellite the ACE spacecraft is located at the libration point L1 at a distance of $\sim 1.5$ million $\mathrm{km}$ from the Earth. Geomagnetic index data were obtained from World Data Center C2 for Geomagnetism, Kyoto [http://wdc.kugi.kyoto-u.ac.jp/].

\section{Results of observations}

\subsection{Geomagnetic and radiation conditions in NES during May $19-28,2021$}

During the period May 19-28, 2021, significant variations of the NES radiation associated with solar activity were detected. Experimental data from three spacecraft, i.e. Meteor, Electro and Arktika located at different orbits, as well as data on the solar wind and geomagnetic activity parameters, allow reliable diagnostics of the state of not only the Earth's magnetosphere, but also of the interplanetary medium.

The geomagnetic environment in OKP on May 19-28, 2021 can be characterized as moderately disturbed. Two magnetic storms were observed with a minimum $\mathrm{D}_{\text {st }}$ variation of $-34 \mathrm{nT}$ at 20.05 and $-28 \mathrm{nT}$ at 27.05 (Fig. 1). The storms were caused by the arrival of interplanetary structures to the Earth, characterized by an increase in the SW velocity and the southern component of the IMF. As a result of a weak storm on 20.05, an increase in the flux of relativistic electrons occurred, which can be seen from geostationary satellites (Electro-L2 and GOES-16) data, see Fig. 1, as well as in polar orbit (SC Meteor-M2), see Fig. 2. The second storm on May 27 led to a drop in the flux of relativistic electrons. Dynamics of the relativistic electron fluxes at the maximum of the outer radiation belt according to data from a polar orbit is shown in Fig. 3.

For radiation monitoring, together with the ERB electron component, the proton component is also an important factor. The radiation belt structure when the Arktika-M1 satellite passes through it with the indication of the L-parameter values can be seen in Fig. 4. Differences in the flux magnitudes are determined by the particle flux pitch-angle distribution. 


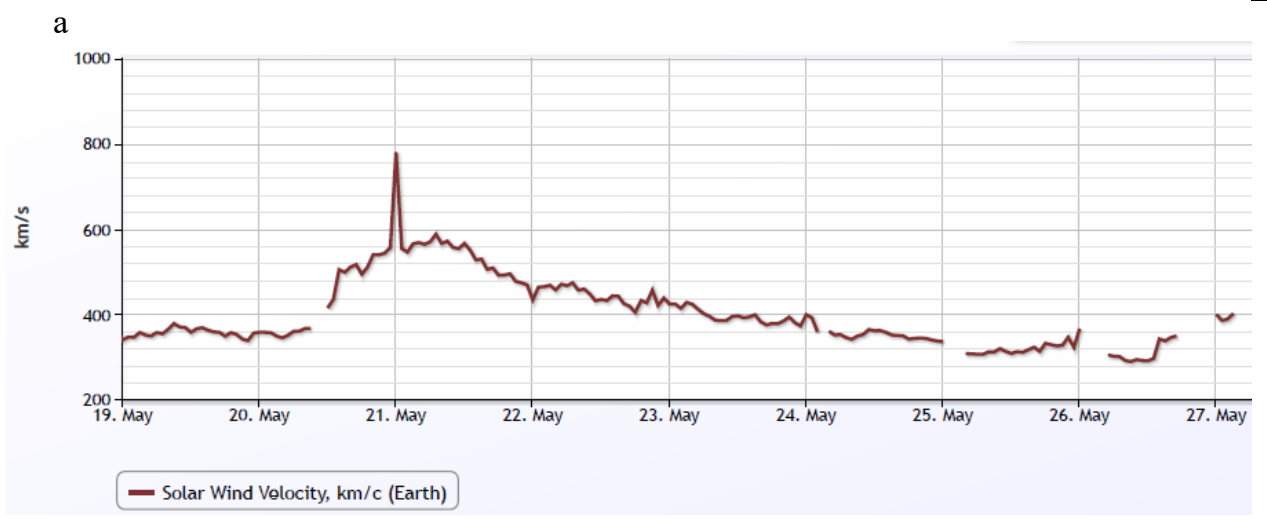

b
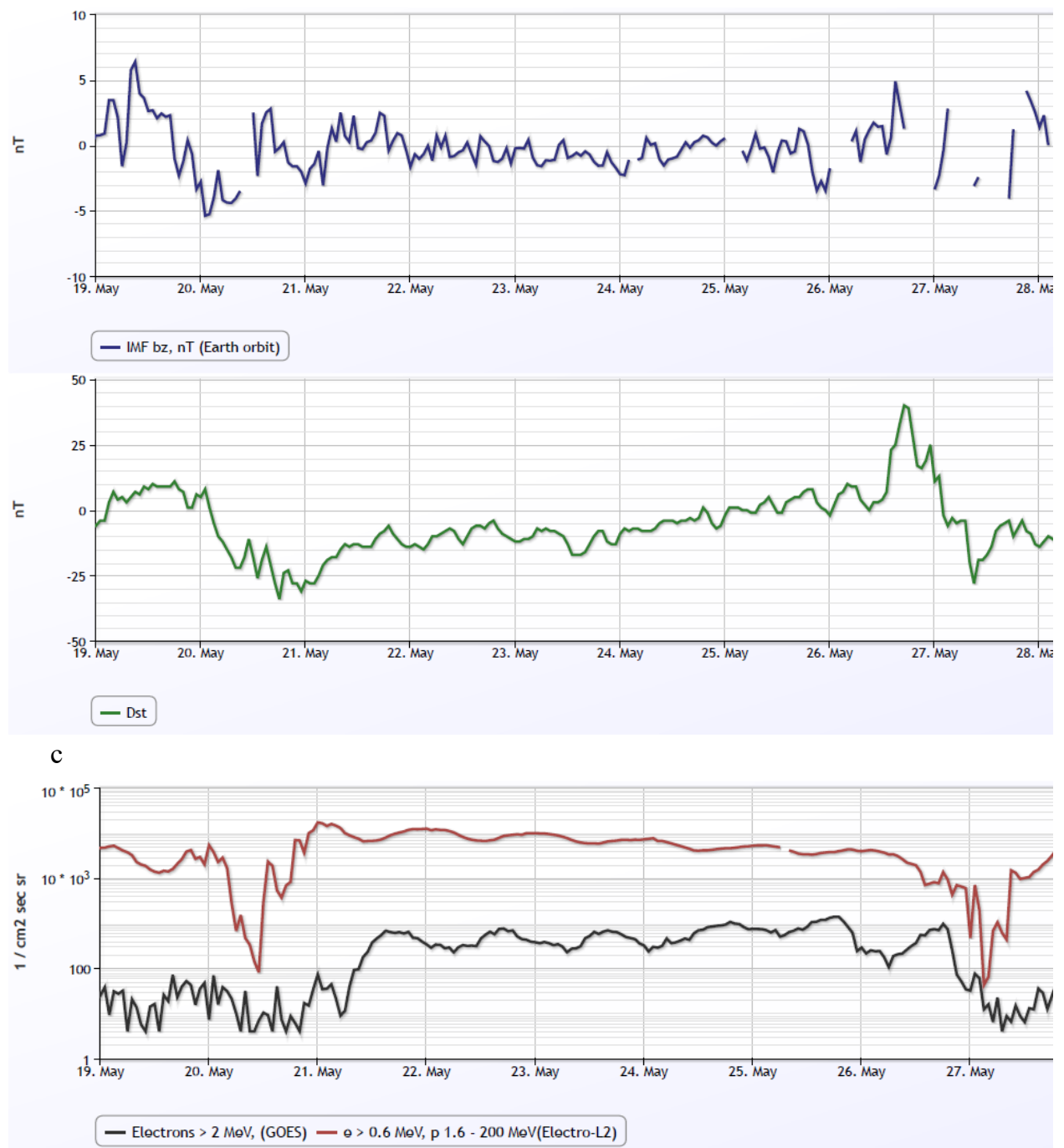

Fig. 1. Time profiles 19-28.05.2021 of SW velocity (a), interplanetary magnetic field (IMF) $B_{Z^{-}}$ component (b, upper panel), $\mathrm{D}_{\mathrm{st}}$-variation ( $\mathrm{b}$, low panel), ERB electron fluxes at geostationary orbit (c). 

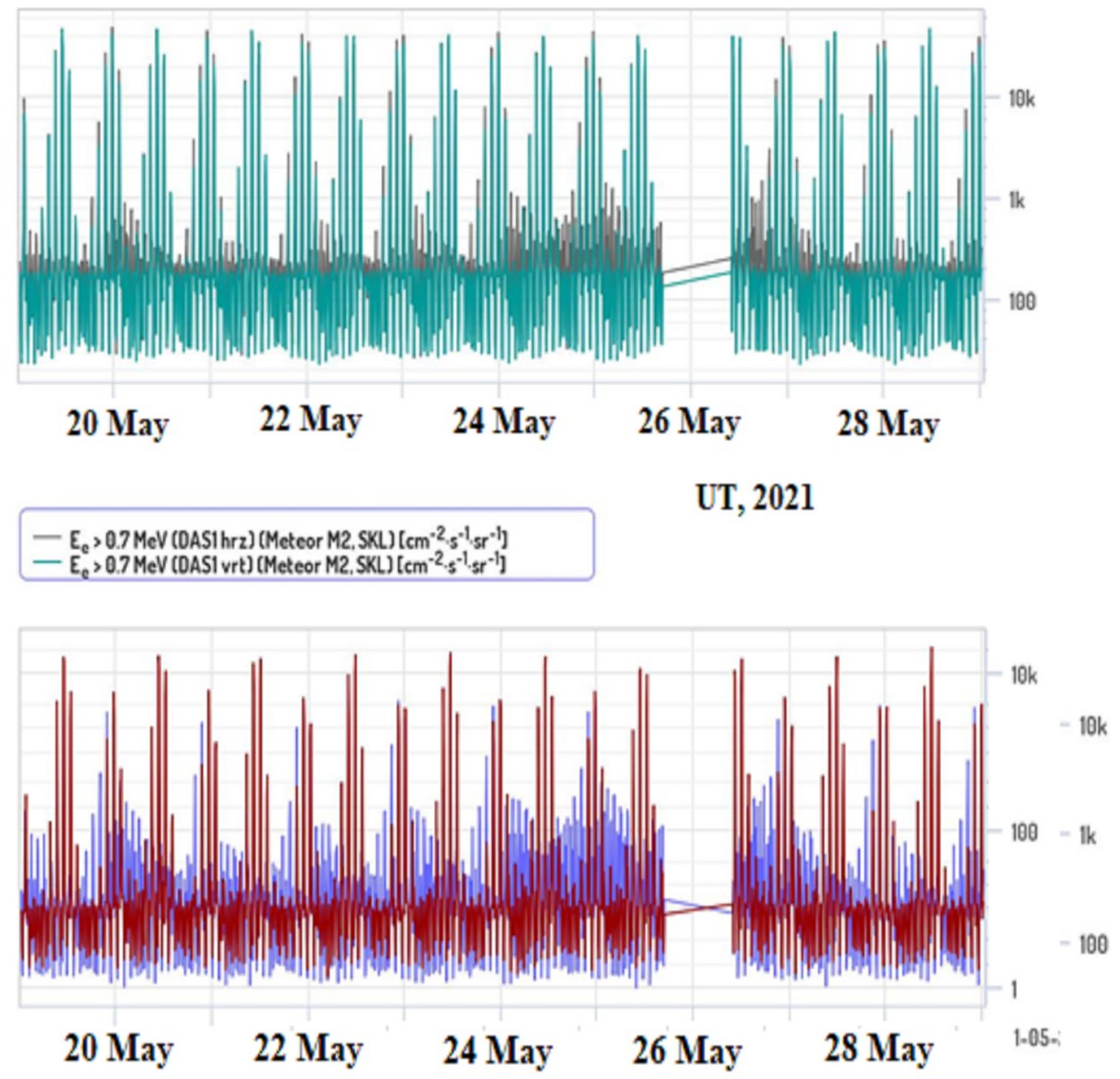

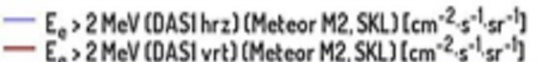

UT, 2021

Fig. 2. Time profiles of the ERB electron fluxes in the ranges $>0.7 \mathrm{MeV}$ (upper panel) and $>2 \mathrm{MeV}$ (bottom panel) obtained from polar orbit 19-28.05.2021.

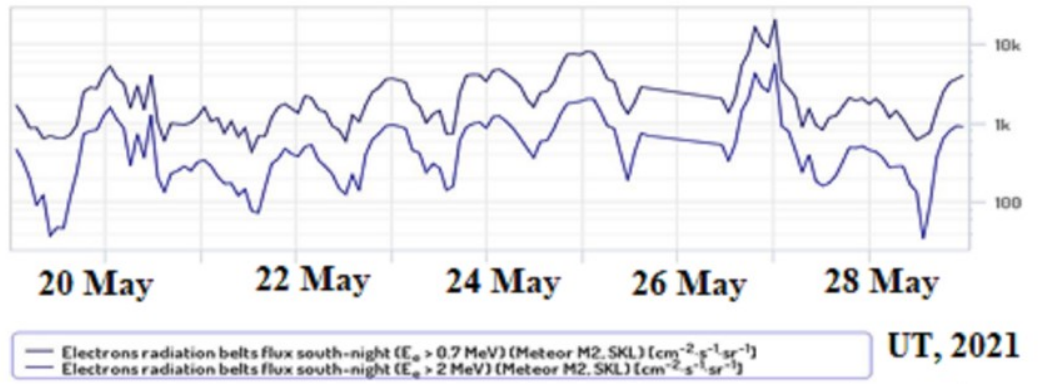

Fig. 3. Time profiles of electron fluxes at ERB maximum obtained from polar orbit data 1928.05.2021. 


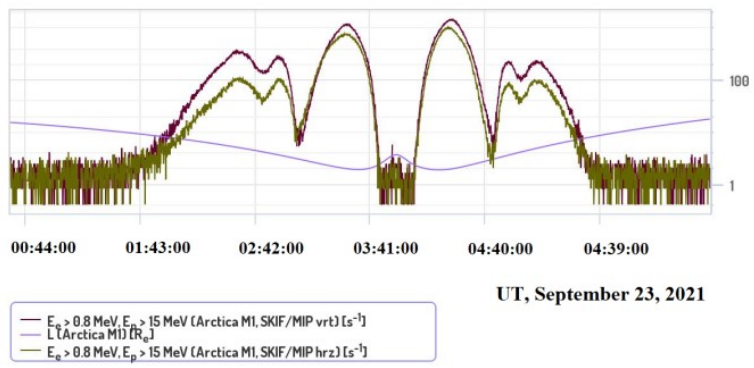

Fig. 4. Space-time profile of the ERB $\left(E_{\mathrm{e}}>0.8 \mathrm{MeV}, E_{\mathrm{p}}>15 \mathrm{MeV}\right.$ on the Арктика-M1 SC orbit 23.05.2021 in 00-06 UT.

\subsection{Solar proton events during September $04-10,2017$}

One of the tasks of the experiment with SKIF instrument is the detection of energetic charged particles during solar proton events (SPE). On September 04-10, 2017, three series of powerful flares occurred on the Sun. In the first series on September 4, at 20:30 UT, a flare of the maximum M5.5 class was detected, in the second, on September 6, at 12 UT, an X9.3 class flare, and in the third, on September 10, an X10 class flare. The latest flare is the most powerful X-ray flare that has occurred on the Sun in the past 12 years.

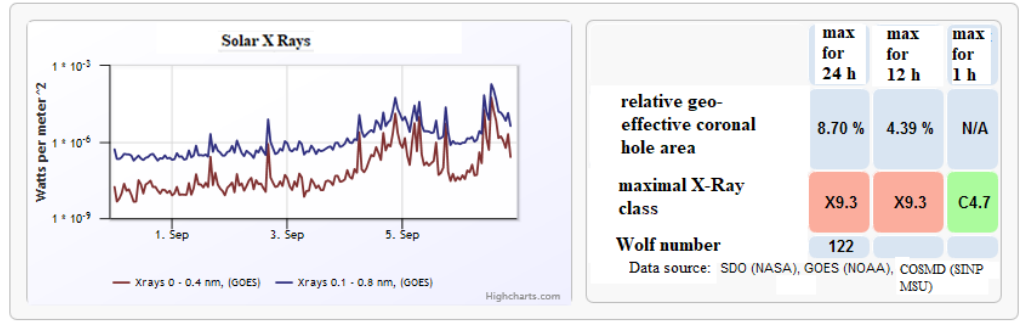

Fig. 5. Presentation of solar activity data on COSMD website for September 06, 2017.

The website of the Center for Operative Space Monitoring Data (COSMD) provides daily data on solar activity observations [http://swx.sinp.msu.ru/apps/geospace_now.php?gcm=1]. The power of X-rays from the Sun is shown according to the data of the NOAA GOES SC data, and the area of coronal holes is presented according to the SDO SC data. The data on the X-ray emission of the Sun in September 2017 were obtained from the GOES-15 spacecraft (see Fig. 5).

The dynamics of the solar X-ray power and the SW velocity, as well as the solar proton fluxes according to the geostationary satellite Electro - L2 and GOES - 15 data is shown in Fig. 6. The result of the first series of flares was a solar protons flux increase on September 04-08, 10-16, 2017, registered in the NASA SPE catalog [https://umbra.nascom.nasa.gov/SEP/seps.html] as one event, while in the catalog, presented on the COSMD website [http://swx.sinp.msu.ru/apps/sep_events_cat/index.php?gcm=1\&lang=ru], this period is considered as several separate SPE, as it is associated with various solar phenomena [5]. It can also be seen that the solar proton flux propagated in the disturbed interplanetary medium (Fig. 6b). The complex time profile of particle fluxes is associated with these factors (Figs. 6c and 6d). The increase in solar proton fluxes on September 10 is one of the strongest SPEs of the 24 solar activity cycle, the second GLE and the last event of this cycle. 
$\mathrm{a}$

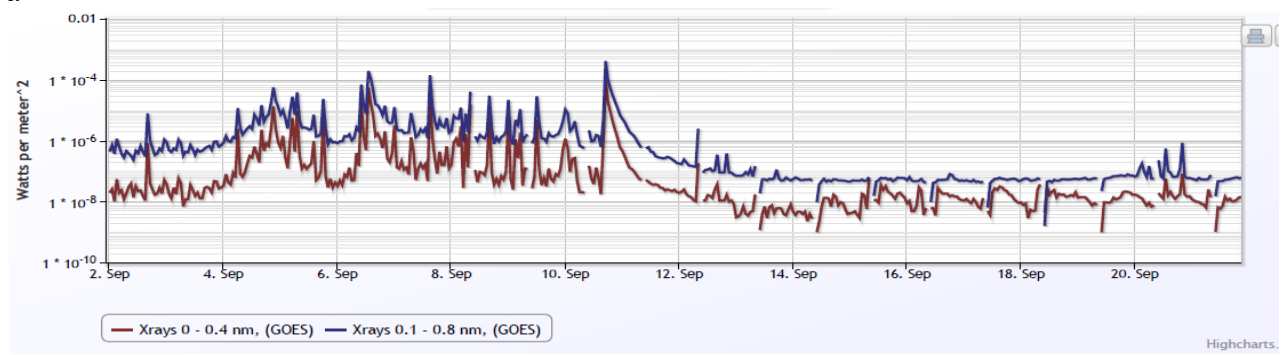

$\mathrm{b}$

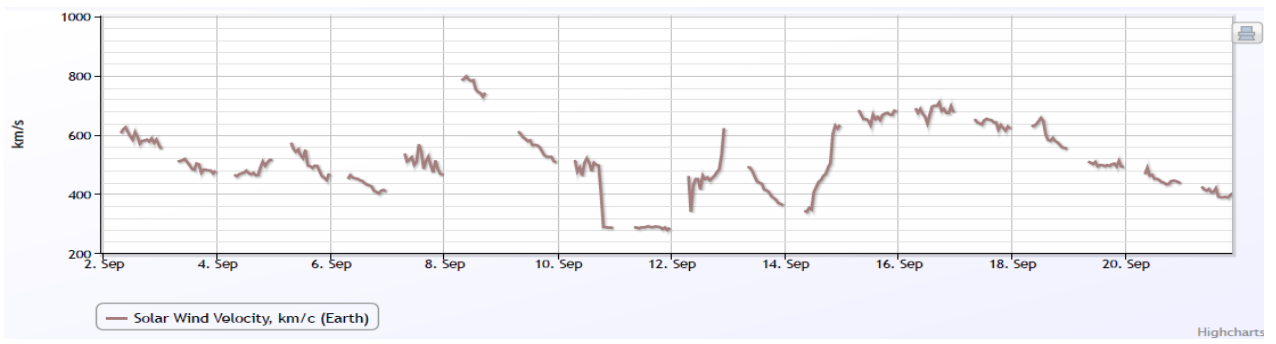

$\mathrm{c}$

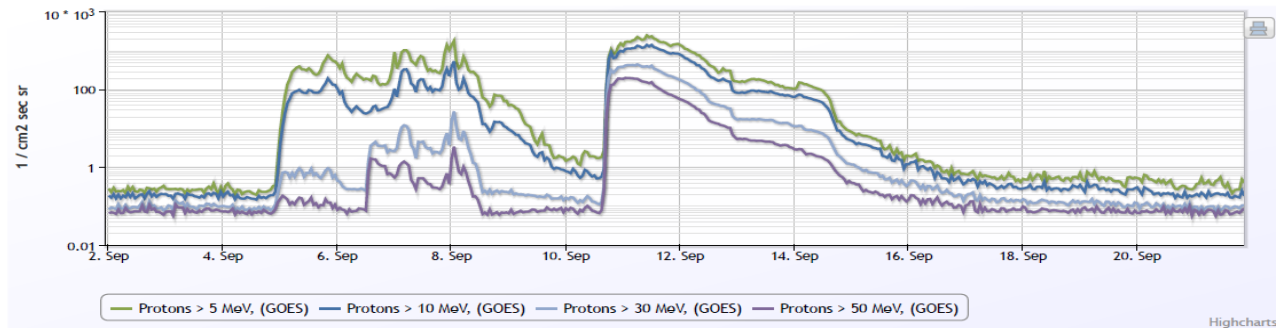

d

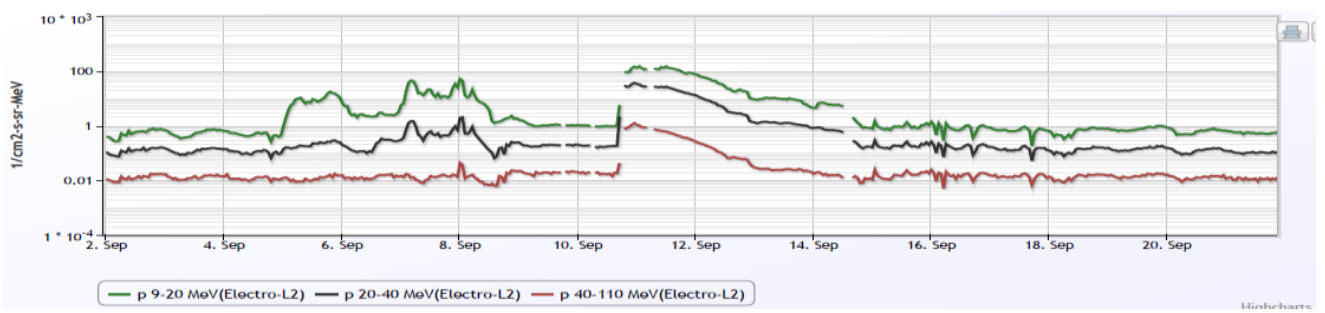

Fig. 6. Time profiles of solar X-ray power (a), SW velocity (b) and solar proton fluxes on the geo-stationary orbit (c, d) $02.09-20.09 .2017$. 


\subsection{Solar proton events during May 28, 2021}

The solar proton event on May 28, 2021 is one of the first SPEs of the $25^{\text {th }}$ solar activity cycle. The event is weak, despite this, the flux of solar protons was detected on all spacecraft on which the SKIF instrument was installed.
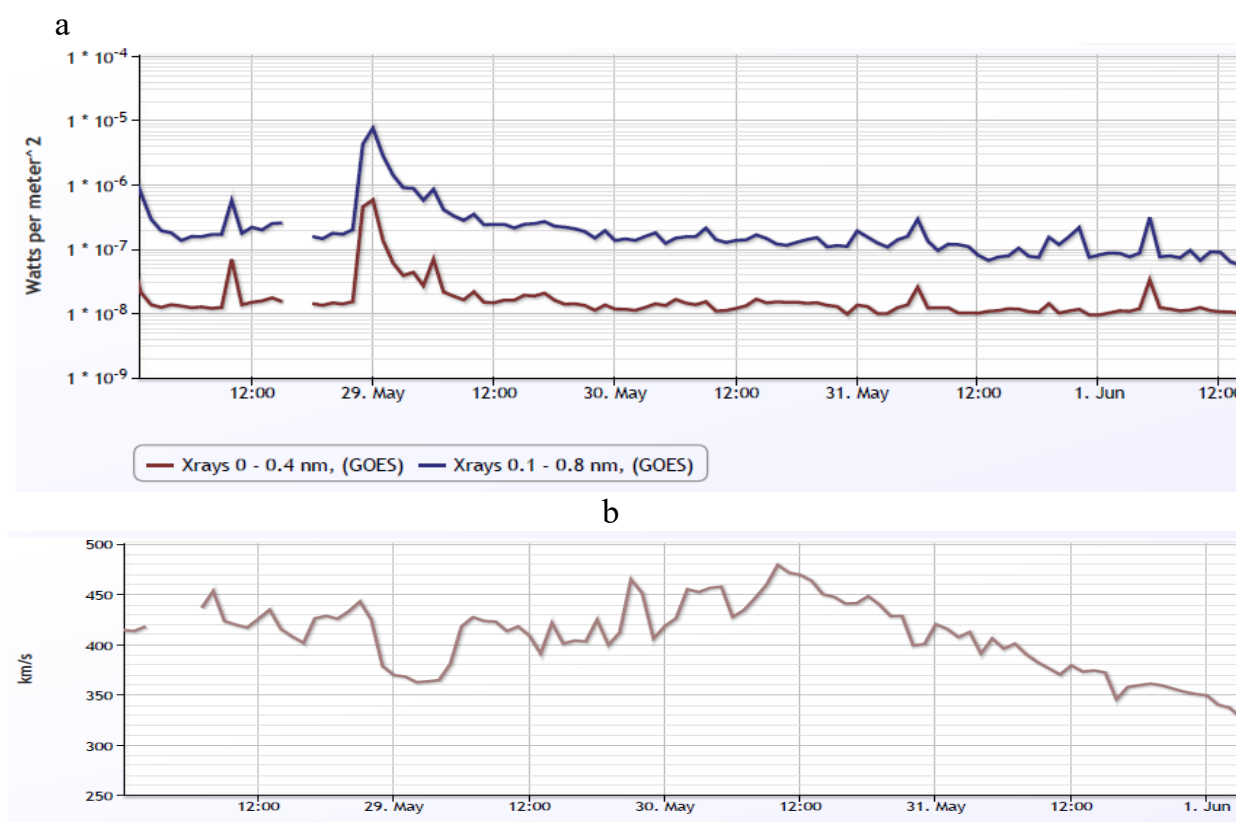

- Solar Wind Velocity, km/c (Earth)

C

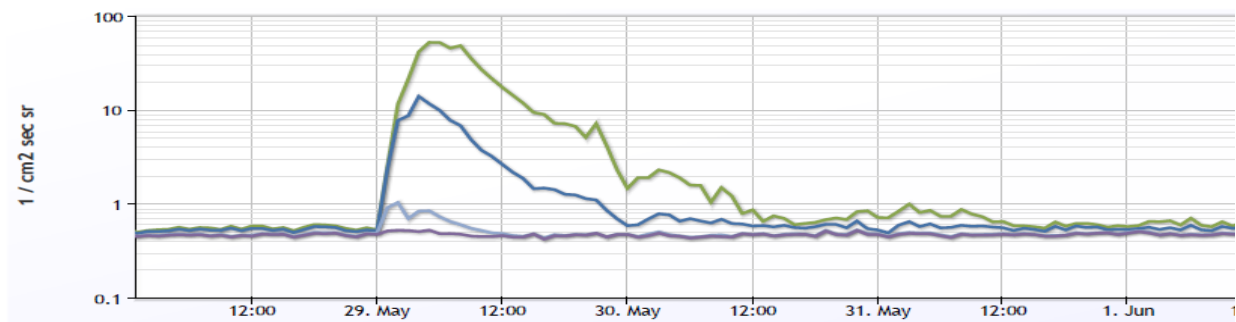

- Protons > $5 \mathrm{MeV}$, (GOES) - Protons > $10 \mathrm{MeV}$, (GOES) - Protons > $30 \mathrm{MeV}$, (GOES) - Protons > $50 \mathrm{MeV}$, (GOE d

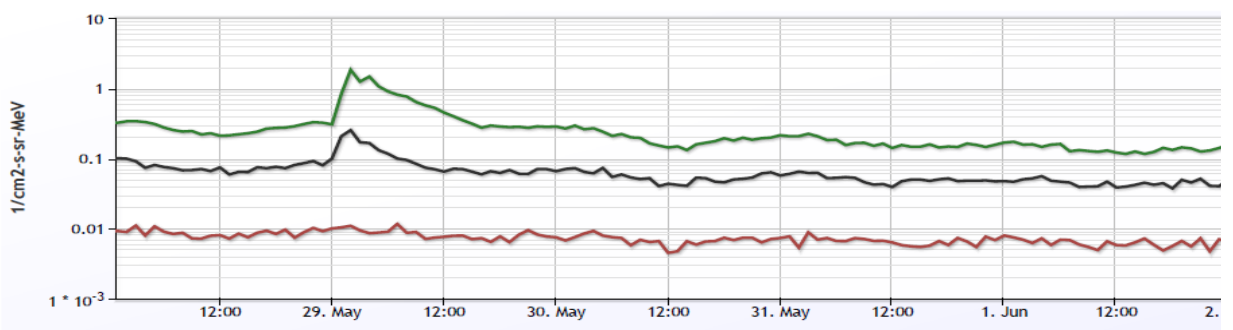

- p 9-20 MoV(Electro-L2) - p 20-40 MoV(Electro-L2) - p 40-110 MoV(Electro-L2)

Fig. 7. Time profiles of solar X-rays (a), SW velocity (b) and solar proton fluxes at geostationary orbit (c, d) during $28.05-02.062021$. 


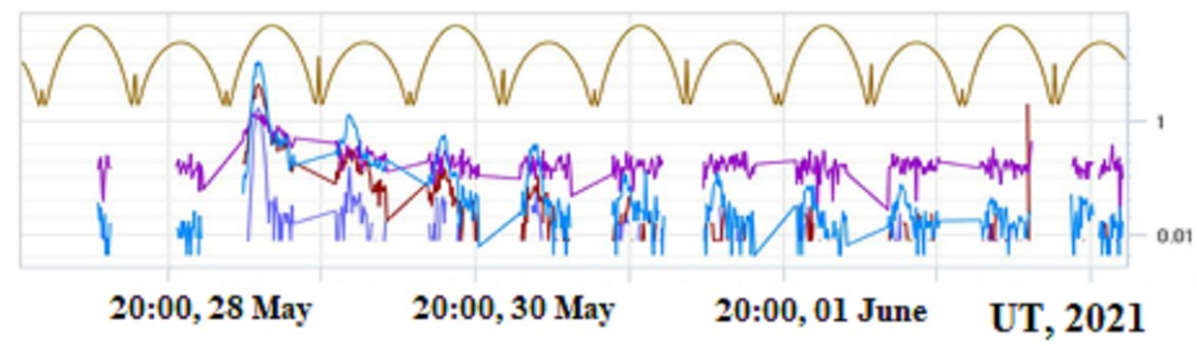

\begin{tabular}{|c|c|}
\hline 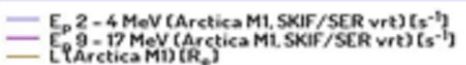 & 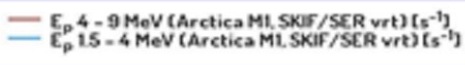 \\
\hline
\end{tabular}

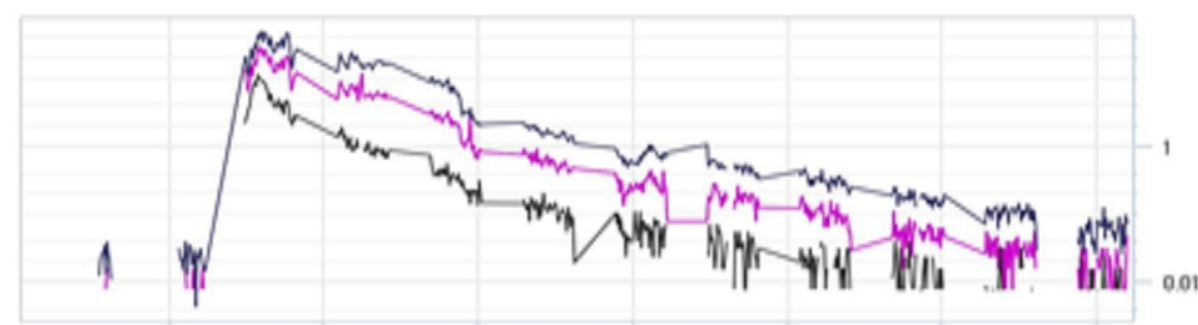

\section{0:00,28 May 20:00,30 May 20:00,01 June UT, 2021}
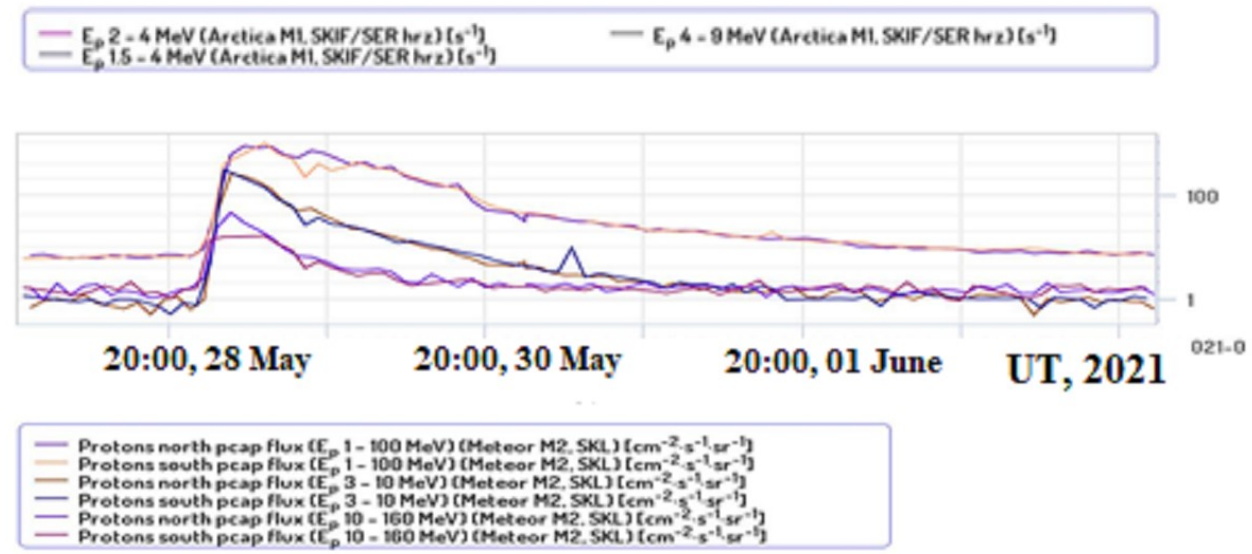

Fig. 8. Time profiles of solar proton fluxes during $28.05-02.062021$.

An increase in solar proton fluxes on May 28, 2021 is associated with an X-ray class C9.5 flare on the Sun (see Fig. 7) at 22:19 UT with helio coordinates N18W67 in the active region (AR) 12824. The flare occurred at the western longitude of the solar disk, and therefore the time profiles of solar proton fluxes have a rather steep leading edge (Figs. 7c and $7 \mathrm{~d}$ and Fig. 8). Solar protons propagated in the interplanetary medium inside a highspeed solar wind flow (Fig. 7b); therefore, the time profile of the fluxes is rather smooth. The SPE is weak, i.e. the maximum flux of protons with energies $>10 \mathrm{MeV}$ was (according to the GOES satellite data, Fig. 7c) was $\sim 13.9 \mathrm{pfu}$, where $1 \mathrm{pfu}=1 \mathrm{particle} /\left(\mathrm{cm}^{2} \cdot \mathrm{s} \cdot \mathrm{sr}\right)$. The time profiles of solar protons detected inside the magnetosphere in the region of closed geomagnetic field lines (data from the Electro and GOES-16 satellites) is shown in Figs. 7c and $7 \mathrm{~d}$. The time profiles according to the Meteor - M2 satellite data in the Earth polar cap areas and the Arktika - M1 satellite data on the magnetic field force open lines and partially 
outside the magnetosphere are shown in Fig. 8. The data from polar low-orbit SC make it possible to substantially supplement the data about SCR events received from geostationary satellites. The proton flux profiles according to the Meteor - M2 satellite data obtained separately over the Earth northern and southern polar caps are shown in bottom panel of Fig 8 . The asymmetry of the particle arrive is practically not detected in this event.

\section{Conclusion}

The first results of energetic charge particle flux variations in NES from multi-satellite observations at different altitudes are presented. Such observations give a relatively complete picture of electron and proton flux dynamics at different areas, including highlatitude crossings of ERBs at altitudes about $800 \mathrm{~km}$ (Meteor SC), the edge of outer electron belt at geo-stationary orbit $(36000 \mathrm{~km}$, Electro-L SC) and polar cap at $600 \mathrm{~km}$ altitude as well as the exit to the interplanetary space $(39000 \mathrm{~km}$, Arktika - M1 SC). The results of complex measurements are presented for different kinds of NES radiation environment disturbances, such as geomagnetic storms caused by solar activity (events 1928.05.2021), and solar proton events 04-10.09.2017, 28.05.2021. These observations indicate on that all kinds of geo and helio- activity lead to significant variations of energetic charge particle flux variations and should be monitored for radiation condition forecasting.

\section{References}

1. M.I. Panasyuk, et al., Model cosmosa. T.1. Physicheskie uslovija v kosmicheskom prostranstve (Russ.) (KDU, Moskva, 2007)

2. O.P. Verkhoglyadova, C.D. Bussy-Virat, A. Caspi, et al., Space Weather, 18 (2020)

3. M.I. Panasyuk M.I., M.V. Podzolko, V.V. Kalegaev, et. al., Moscow University Physics Bulletin 73, 687 (2018)

4. V. Kalegaev, M. Panasyuk, I. Myagkova, et al., J. Space Weather and Space Climate, 9 (2019)

5. G.A. Bazilevskaja, E.I. Daybog, Yu.I. Logachev, et al., Geomagnetizm i aeronomia (Russ.) 61, 8 (2021). 